\title{
Bioactivity of Whey Peptides
}

\author{
Tolulope J. Ashaolu1, Joseph 0. Ashaolu2 ${ }^{2 *}$ \\ ${ }^{1}$ College of Food Science, Southwest University, Chongqing, P.R. China \\ ${ }^{2}$ International Health Program, School of Medicine, National Yang-Ming University, Taipei, Taiwan \\ E-mail: ashaolut@gmail.com; noblebidemee@gmail.com \\ *Corresponding author details: Joseph O. Ashaolu, noblebidmee@gmail.com
}

\begin{abstract}
Numerous studies on whey protein have proven its nutritional and physiological functions. Upon enzymatic hydrolysis, preferable to other means of hydrolysis like fermentation or physico-chemical methods, beneficial bioactive peptides are generated. The use of whey protein in diverse sectors of the food manufacturing industry does not stifle the production of its novel multiple products such as whey peptides, reported to confer multifarious biological activities based on wide ranges of research and indepth investigations. The present review considers an overview of whey peptide production, and their popularly reported bioactivities. Some precautions to be taken amongst other recommendations were also discussed.
\end{abstract}

Keywords: whey protein; enzymatic hydrolysis; bioactive peptides; physico-chemical; physiological functions

\section{INTRODUCTION}

In the dairy industry, cheese production leads to the production of certain proteinaceous byproducts including whey protein is found. It is that watery liquid with yellow/green to bluish tinge color, produced from cows', goats', sheep's, and even camels' milk.

The use of whey protein spans over 3000 years, and has become a highly marketable health product with several claims associated with it. Being an excellent source of nutrients such as vitamins, lactose and bioactive peptides, the present study therefore, takes a dive into an overview of whey proteins functions, the production of whey peptides, and popular bioactivities including antihypertensive, antioxidant, immunomodulatory and antimicrobial, cardiovascular among other miscellaneous properties.

\section{WHEY PROTEINS}

The commonest types of whey obtained from milk production are acid and sweet. The commonest type of the two is sweet whey. The whey proteins represent 15$20 \%$ of total milk proteins, constituting about $0.7 \%$ $(\mathrm{w} / \mathrm{v})$ of the total solid content of whey obtained from milk [1]. Several other minor proteins can be found in whey, including lactoperoxidase (LP), lactoferrin (LF) and proteose-peptone (PP). There are also other major proteins found, including $\beta$-lactoglobulin $(\beta-L G), \alpha-$ lactalbumin ( $\alpha$-LA), bovine serum albumin (BSA), and immunoglobulins (IGs) [2]. The proteins are globulated, capable of denaturation by heat, insensitive to calcium ions, and highly soluble. To attain these, their internal cystein-sulfhydryl bridge is used to form intramolecular bonds [3].

The largest whey protein is BSA $(1.5 \%)$ based on its molecular structure and weight $(66,399 \mathrm{kDa})$, followed by IgG, which comprises of four polypeptide chains and is $1 \%$ of the total protein content in milk [4]. These composition, structure, weight and size parameters are important to study whey protein's functional properties such as emulsification, foaming stability, gelation, sensory and hydration properties [1].
Whey proteins also have more nutritional values than most other consumed plant and animal based proteins like egg, soy and casein proteins because they possess the essential, branched and sulfur-containing amino acids of paramount importance in the regulation of multifarious antioxidative, metabolic functions, blood glucose homeostasis, satiating effects, body-weight control and obesity treatment among other functions $[3,5]$.

Cysteine derived from $\beta$-LG actively participate in glutathione (GSH) production, which is important in immunomodulation, improved liver functioning, cancer and Alzheimer suppression [6]. Infants formulas are often enriched with $\alpha$-LA since it contains high amounts of tryptophan, which serves as a precursor of serotonin meant to aid sleeping mode and "feel good" experience [7]. Lactoferrin (LF) protect the mammary glands and the gut via provision of vast nutrients, minerals and amino acids capable of mitosis and enhanced immunity.

Lactoferrin has the capacity to bind to iron thus enabling it to provide antifungal, antibacterial and antiviral effects [8].

Immunoglobulins and Lactoperoxidase have been reported to enhance gut health, demonstrate therapeutic and neonates protection, as well as antimicrobial effects, respectively. These whey proteins exert better performance when broken into shorter peptides by in vitro or in vivo digestion.

\section{WHEY BIOACTIVE PEPTIDES PRODUCTION}

Peptides are potent and inactive within their parent proteins only to be activated upon enzymatic, fermentative, or gastrointestinal digestive processes [9, 10]. Bioactive peptides have between two to twenty amino acids in their primary sequence, which are always implicated in their activities. The ratio of specific enzyme used to whey proteins will determine factors like composition, hydrophobicity of the C-terminal, molecular mass and order of the amino acids in the peptide sequences, which then impacts their bioactivities including immunomodulatory, antihypertensive, hypolipidemic, antimicrobial, antidiabetic, anticarcinogenic and antioxidant effects [11]. Recently bioactive whey peptides 
were employed in fabrication of nutraceutical-confining particles [12].

Several hydrolysis or digestion methods may be used to release whey bioactive peptides from their parent proteins but enzymatic hydrolysis is preferred to solvent extraction, fermentative or chemical hydrolysis based on its absence of toxic residual chemicals or organic solvents in the hydrolysates and peptides [9]. The enzyme must be specific and other conditions of enzymatic hydrolysis including $\mathrm{pH}$, temperature, hydrolysis time and enzyme/substrate ratio, be optimal for the production of whey bioactive peptides because after treatment with several enzymes, a mixture of peptides with broad specificity become available in the hydrolysate pool. The pool is passed through a purification process in order to isolate bioactive peptides with specific bioactivity [9]. The sequence of amino acids and their overall chain length become altered after digestion of their parent protein by proteolytic enzymes such as chymotrypsin, pepsin, papain, peptidase and trypsin, into smaller peptides and free amino acids by breaking their peptide bonds [13]. The degree of hydrolysis (DH) will also affect the type, size, and amino acid contents of the peptides produced. If the whey protein is extensively hydrolysed, more free amino acids become available rather than bioactive peptides, thus the enzymatic reaction must be regulated.

The obtained whey hydrolysates are purified, and the peptides are identified based on fractionation methods that utilize molecular size principle, such as gel chromatography and membrane filtration, or the principle of hydrophilic/hydrophobic interactions as found in reverse phase chromatography. The charges on the protein molecules can also be used in screening the whey hydrolysates, as found in the use of ion-exchange chromatography. Gel filtration is a fractionation technique whereby a packed gel in a buffered column allows the migration of purified peptides without affecting their activities [9]. The technique is applicable to proteins originating from diverse sources. Chromatography may be synergized with several other techniques according to charges, hydrophilicity or hydrophobic interactions, $\mathrm{pH}$, adsorption or metal-binding properties $[9,14]$, after which identification is carried out with mass spectrometry (both liquid and tandem) and other bioinformatic tools.

\section{BIOLOGICALS AVTIVITIES OF WHEY PEPTIEDS}

Table 1 contains a selected list of the most recent whey peptides with their biological activities. The most popular reported biological functions of whey peptides are as follows:

\section{Antimicrobial function}

Certain milk peptides and whey lactoferrin (lactoferricin and lactoferrampin) have been reported to possess antimicrobial properties against Gram-positive and Gramnegative bacteria (e.g. Bacillus subtilis, Escherichia coli, and Pseudomonas aeruginosa), yeast, and fungi [15]. The antimicrobial peptides are less than $10 \mathrm{kDa}$, composed of basic, aliphatic and polar amino acids. They are amphipathic, cationic, and have isoelectric point greater than 10 [16]. Antibacterial, antifungal and antiviral activities are common with lactoferricin peptides due to their ease of forming amphipathic structures. For instance, the glycomacropeptide, $\beta$-LA and $\alpha$-LA derived peptides have shown inhibition to Gram positive bacteria [17]

The action mechanism of antimicrobial whey peptides involves some sort of electrostatic interactions whereby the positively charged peptides reacts with the anionic cell surfaces in order to interfere with membrane integrity and permeability via blockage of calcium and magnesium sites [18].

\section{Antioxidant function}

The amphiphilicity of several peptides enables them to possess antioxidative property because a fine balance is obtained between the hydrophilic and hydrophobic segments. Oxidants may be endogenous or exogenous, and both reactive oxygen species (ROS) and reactive nitrogen species (RNS) as the commonest causal radicals of oxidative stress, as well as chlorine $(\mathrm{Cl})$, bromine $(\mathrm{Br})$ and sulphide (S) reactive species [9].

Oxidative stress is a result of an imbalance between the antioxidant defence mechanism and the amount of oxidants produced, which then endangers the integrity of proteins and cells within the human systems leading to quick aging, cardiovascular and chronic diseases. Thus, molecular compounds effective against oxidation reactions are always on demand and food scientists have lived up to this. Digestion of food proteins could lead to inhibition of this oxidative process. Artificially synthesized antioxidants including butylated hydroxyanisole and butylated hydroxytoluene are often utilized for the inhibition of lipid oxidation in food and biological systems except for certain harmful activities, which paved the way for natural antioxidative compounds from plants and animal sources [5].

Tryptophan, histidine, proline and tyrosine are the usual hydrophobic residues found in antioxidative peptides isolated from milk proteins, which constitute between five to eleven amino acids [2]. Sulfur-containing amino acid residues and aromatic R-groups intervene in antioxidative activity of peptides via various mechanisms such as free radical scavenge, pro-oxidative transition metals chelation, hydroperoxides reduction, and food texture modification [12].

The in vitro and in vivo mechanisms associated with antioxidative peptides like the antioxidant response elements (AREs), HAT (hydrogen atom transfer), SET (single electron transfer) mechanisms, or the activity-based mechanisms of red-ox enzymes such as glutathione peroxidase (GPx), peroxiredoxins, superoxide dismutase (SOD), catalase (CAT), glutaredoxin (Grx) and glutathione reductase, have been reported [9].

Using a selection of various pancreatic enzymes, HernándezLedesma et al. [19] reported that whey peptides derived from $\beta$-LG and $\alpha$-LA, especially the sequence Trp-Tyr-SerLeu-Ala-Met-Ala-Ala-Ser-Asp-Ile isolated from $\beta$-LG by Corolase PP demonstrated higher oxygen radical scavenging activity than butylated hydroxyanisole, based on their molecular weights $(0.1-2.8 \mathrm{kDa})$.

\section{Anti-hypertensive function}

Cardiovascular diseases are linked to coronary heart disease, congestive heart failure, stroke, renal disease, and high blood pressure. These diseases affect a quarter of the human population. Brandelli et al. [2] reported that anti-hypertensive whey peptides are the most relevant and studied peptides due to their importance in high blood pressure reduction.

Many bioactive peptides have been designed to inhibit angiotensin converting enzyme (ACE) so that angiotensin I would not be converted to angiotensin II. The functions of renin angiotensin-I enzyme (ACE) is to regulate peripheral blood pressure and convert decapeptide angiotensin-I to octapeptide angiotensin-II among others. The angiotensinII is an effective vasoconstrictor. The ACE also catalyses the breakdown of nonapeptide bradykinin, a vasodilator [12]. The ACE inhibitory peptides fall within the range of two to twelve amino acids, with the C-terminals of proline, tyrosine, tryptophan and phenylalanine being the most ACE active sites bonders for the purpose of orchestrating their inhibitory potentials.

Glycomacropeptide and certain peptides synthesized from $\alpha$-LA and $\beta$-LA using plant-derived enzymes have 
demonstrated excellent ACE-inhibitory activity in vitro, and in simulated gastrointestinal digestion [20]. In some studies, $\alpha$-lactorphin (YGLF) prepared from $\alpha$-lactalbumin, as well as $\beta$-Lactorphin (YLLF) and $\beta$-lactotensin (HIRL) prepared from $\beta$-lactoglobulin, showed blood pressure reduction in spontaneously hypertensive rat models, as YLLF showed the highest ACE-inhibition in comparison with YGLF and HIRL [21, 22].

However, in vivo anti-hypertensive peptide mechanism is not yet clear despite the identification of anti-hypertensive whey peptides in vivo.

\section{Immunomodulatory function}

Whey peptides have demonstrated immunomodulatory effects at the levels of both adaptive and innate immune responses as found in the activation and proliferation of lymphocytes, regulation of cytokines, production of antibody, increased phagocytic ability of macrophages, and stimulation of immunoglobulin generation [11].

Due to their structure-function relationship, the sequence, charge, length, molecular structure, hydrophobicity and amino acids composition of the peptides will describe their immunomodulatory potentials. They are often hydrophobic with 2-10 amino acids contents. Their action mechanisms also remain unclear, but with certain speculations involving the interactions of molecules via chemical bonding between peptides and receptors on the immune cells surfaces $[11,23]$.

Studies on whey $\alpha$-LA and $\beta$-LG hydrolysates fermented with lactobacillus paracasei showed their immunomodulatory activity including suppression of lymphocyte stimulation, upregulation of IL-10, and downregulation of proliferating splenocyte. They also act in the downregulation of INF- $\gamma$ and IL-4 secretion in murine models [3]. In a pig study, their mucosal immunity were enhanced by the increased levels of immunoglobulins $\mathrm{A}, \mathrm{G}$ and $\mathrm{M}$ (IgA, IgG and IgM) upon supplementation of the pigs diet with whey-derived peptides, lactoferricin and lactoferrampin [24]. Of these two peptides, lactoferricin showed its blocking activity of interleukin-6 (IL-6), implicated in rheumatoid arthritis inflammation [25].

Furthermore, in other studies, whey peptides have demonstrated improved lymphocyte proliferation, blocked pro-inflammatory cytokine IL-8 release, decreased ROS levels in mice and induced the production of interleukin-2 (IL-2) and gamma interferon (IFN $\gamma$ ) that generate activation of monocytes and neutrophils $[11,17,26,27]$.

\section{Cardiovascular functions}

Whey peptides also demonstrate hypocholesterolemic, anti-diabetic and anti-obesity biological activities.

Dipeptidyl peptidase IV (DPP-IV) is a crucial peptidase involved in the regulation of infection, growth, digestion, immunity, pain, feeding and many other activities of the body. Inhibition of this enzyme can aid the fight against obesity and diabetes. A simulated gastrointestinal digestion that involved whey peptides were implicated in DPP-IV inhibition in the lower gut, which prevented glucose-dependent insulinotropic polypeptide (GIP) and glucagon-like peptide-1 (GLP-1) from degrading [28]. The half-life of these incretins GIP and GLP-1 become increased in order to stimulate the secretion of postprandial glucosedependent insulin, leading to much more reduced food intake and satiety [12].

In addition, whey hydrophilic peptides and free amino acids were suggested to possess insulinotropic property. Futhermore, whey hydrolysates in synergy with a DPP-IV inhibitory drug (sitagliptin) inhibited DPP-IV, which demonstrated the combinatorial effect of clinical drugs and dietary peptides on Type 2 diabetes therapy [29]. Moreover, DPP-IV inhibitory peptides derived from $\beta$-LA were reported to decrease blood glucose level in in vitro and in vivo studies $[30,31]$.

Whey peptides cholesterol-lowering activity was also demonstrated in rat studies whereby consumption of $\beta$ LA derived peptides led to a higher amoiunt of fecal steroids as against casein hydrolysates, indicative of chelation of taurocholate or enhanced micellar cholesterol solubility [32].

Likewise, consumption of 30 grams of whey peptides drink for two years was linked to weight-derived hepatic steatosis reduction in elderly women [33].

For obesity and diabetes, more than half billion population of the world have been trapped and thus need some pragmatic help, what they consume. Food proteins and other food products are preferred to anorectic drugs based on satiety, safety and loss of weight.

\section{Miscellaneous functions}

Antithrombotic, antiviral, opioid, anticancer, and mineralbinding peptides are among other reported functions of whey peptides $[2,6]$. Lactoferricin and its derived peptides have been highly antithrombotic, antitumor, antiinflammatory and antiviral, while $\beta$-casomorphins produced from $\alpha$-casein and $\beta$-lactorphins produced from $\alpha$-LA and $\beta$-LG, respectively, have shown antihypertensive, immunomodulatory, opioid and cytomodulatory activities [34]. Furthermore, iron-binding abilities and antigenic properties were associated with heat-induced whey peptides [3]. So many of the bioactive peptides derived from whey proteins are multifunctional.

Some bioactive whey peptides have been subjected to Maillard reaction for possible improvements in developing novel valorised foods [35]. The amino groups of proteins and carbonyl groups of reducing sugars can form a linkage via a Maillard reaction [36], and browning may occur in the process. Thus, the main drawback of this idea is that carcinogenic substances such as acrylamide, and antinutritional compounds are formed from this reaction.

\section{OTHER CONSIDERATIONS AND CONCLUSIONS}

The safety of whey bioactive peptides can not be overemphasized. This safety issue is often associated with their production methods. Thus, the production conditions such as the hydrolysis, purification and identification conditions need detailed and careful control. If considered, a much better uniformity and stability of whey bioactive peptides would be attained. It is suggested that DNA recombination technology may offer more purity for the generated peptides, while nanoencapsulation will aid apt delivery of the bioactive peptides. For commercial production of whey bioactive peptides, toxicity and allergenicity should be considered, including possible harmfulness to certain populations including infants, pregnant women and older adults.

\section{ACKNOWLEDGEMENTS}

This work was self-supported by the authors.

\section{REFERENCES}

[1] Kaur, N., Sharma, P., Jaimni, S., Kehinde, B.A. and Kaur, S., 2020. Recent developments in purification techniques and industrial applications for whey valorization: A review. Chemical Engineering Communications, 207(1), pp.123-138.

[2] Brandelli A, Daroit DJ, Corrêa APF (2015). Whey as a source of peptides with remarkable biological activities. Food Res Int 73: 149-161.

[3] Kareb, 0. and Aïder, M., 2019. Whey and its derivatives for probiotics, prebiotics, synbiotics and functional foods: a critical review. Probiotics and antimicrobial proteins, 11(2), pp.348-369 
[4] Sousa, G. T., Lira, F. S., Rosa, J. C., de Oliveira, E. P., Oyama, L. M., Santos, R. V., and Pimentel, G. D. (2012). Dietary whey protein lessens several risk factors for metabolic diseases: A review, Lipids Health Dis., 11, 67-68.

[5] Mohanty D, Mohapatra S, Misra S, Sahu P (2016) Milk derived bioactive peptides and their impact on human health-a review. Saudi J Biol Sci 23:577583.

[6] Nongonierma A, O'keeffe M, FitzGerald R (2016) Milk protein hydrolysates and bioactive peptides. Adv Dairy Chem Springer, pp 417-482.

[7] Park YW, Nam MS (2015) Bioactive peptides in milk and dairy products: a review. Korean J Food Sci Anim Resour 35:831-840.

[8] Demers-Mathieu V, Gauthier SF, Britten M, Fliss I, Robitaille G, Jean J (2013) Antibacterial activity of peptides extracted from tryptic hydrolyzate of whey protein by nanofiltration. Int Dairy J 28:94101.

[9] Ashaolu, T.J., 2020a. Antioxidative peptides derived from plants for human nutrition: their production, mechanisms and applications. European Food Research and Technology, pp.1-13.

[10] Ashaolu, T.J., 2020b. Applications of soy protein hydrolysates in the emerging functional foods: a review. International Journal of Food Science \& Technology, 55(2), pp.421-428.

[11] Herrera-Ponce, A.L., Alarcón-Rojo, A.D., Salmeron, I. and Rodríguez-Figueroa, J.C., 2019. Physiological health effects of whey protein-derived bioactive peptides: A review. Revista Chilena de Nutricion, 46(2), pp.205-214.

[12] Madadlou, A. and Abbaspourrad, A., 2018. Bioactive whey peptide particles: An emerging class of nutraceutical carriers. Critical reviews in food science and nutrition, 58(9), pp.1468-1477.

[13] Ashaolu, T.J., 2020c. Health applications of soy protein hydrolysates. International Journal of Peptide Research and Therapeutics, pp.1-11.

[14] Sila A, Bougatef A (2016) Antioxidant peptides from marine by-products: isolation, identification and application in food systems. J Funct Foods 21:10-26.

[15] Madureira A, Tavares T, Gomes AMP, Pintado M, Malcata FX (2010) Invited review: physiological properties of bioactive peptides obtained from whey proteins. J Dairy Sci 93:437-455.

[16] Esmaeilpour $M$, Ehsani MR, Aminlari $M$, Shekarforoush S, Hoseini E. Antimicrobial peptides derived from goat's milk whey proteins obtained by enzymatic hydrolysis. J -Food Biosci Technol 2017; 7 (1): 65-72.

[17] Alvarado Carrasco C, Guerra M. Whey as a source of bioactive peptides. An venez nutr 2010; 23 (1): 45-50.

[18] Sah BNP, Vasiljevic T, McKechnie S, Donkor ON. Antioxidative and antibacterial peptides derived from bovine milk proteins. Crit Rev Food Sci Nutr 2016; 58 (5): 726-740.

[19] Hernández-Ledesma B, Dávalos A, Bartolomé B, Amigo L (2005) Preparation of antioxidant enzymatic hydrolysates from $\alpha$-lactalbumin and $\beta$ lactoglobulin. Identification of active peptides by HPLC-MS/MS. J Agric Food Chem 53:588-593.

[20] Tavares TG, Malcata FX (2013) Whey proteins as source of bioactive peptides against hypertension. INTECH Open Access Publisher.

[21] Belem, M. A. F., Gibbs, B. F., and Lee, B. H. (1999). Proposing sequences for peptides derived from whey fermentation with potential bioactive sites. J. Dairy Sci. 82:486-493.
[22] Hernández-Ledesma, B., del Mar Contreras, M. and Recio, I., 2011. Antihypertensive peptides: production, bioavailability and incorporation into foods. Advances in colloid and interface science, 165(1), pp.23-35.

[23] Maestri E, Marmiroli M, Marmiroli N. Bioactive peptides in plant-based foodstuffs. J Proteomics 2016; 147: 104-155.

[24] Tang Z, Y in Y, Zhang Y, Huang R, Sun Z, Li T, Chu W, Kong X, Li L, Geng M, Tu Q. Effects of dietary supplementation with an expressed fusion peptide bovine lactoferricin lactoferrampin on performance, immune function and intestinal mucosal morphology in piglets weaned at age $21 \mathrm{~d}$. Br J Nutr 2009; 101: 998-1005.

[25] Barros de Oliveira CM, Kimiko Sakata R, Machado Issy A, Gerola LR, Salomão R. Cytokines and pain. Rev Bras Anestesiol 2011; 61 (2): 137-42.

[26] Saint-Sauveur D, Gauthier SF, Boutin Y, Montoni A, Fliss I. Effect of feeding whey peptide fractions on the immune response in healthy and Escherichia coli infected mice. Int Dairy J 2009; 19: 537-544.

[27] Badr G, Sayed LH, Omar HE-DM, Abd El-Rahim AM, Ahmed EA, Mahmoud MH.Camel whey protein protects $\mathrm{B}$ and $\mathrm{T}$ cells from apoptosis by suppressing activating transcription factor-3 (ATF-3) -mediated oxidative stress and enhancing phosphorylation of AKT and IкB- $\alpha$ in type I diabetic mice. Cell Physiol Biochem 2017; 41-54.

[28] Nongonierma, A.B.,and FitzGerald, R.J. (2013a). Dipepti dyl peptidase IV inhibitory properties of a whey protein hydrolysate: Influence of fractionation, stability to simulated gastrointestinal digestion and foodedrug interaction. Int. Dairy J. 32:33-39.

[29] Nongonierma, A.B., Gaudel, C., Murray, B.A., Flynn, S ., Kelly, P. M., Newsholme, P., and FitzGerlad, R. J. (2013b). Insulinotropic properties of whey protein hydrolysates and impact of peptide fractionation on insulinotropic response. Int. Dairy J. 32:163-168.

[30] Uchida, M., Ohshiba, Y., and Mogami, O. (2011). Novel dipeptidyl peptidase4 -inhibiting peptide derived from $\beta$-lactoglobulin. J. Pharmacol. Sci. 117:63-66.

[31] Silveira, S.T., Martínez-Maqueda, D., Recio, I., and Hernández-Ledesma, B. (2013). Dipeptidyl peptidase-IV inhibitory peptides generated by tryptic hydrolysis of a whey protein concentrate rich in blactoglobulin. Food Chem. 141:1072-1077.

[32] Hartmann, R., and Meisel, H. (2007). Food-derived peptides with biological activity: From research to food applications. Curr. Opin. Biotech. 18:163-169.

[33] Ooi, E. M., Adams, L. A., Zhu, K., Lewis, J. R., Kerr, D. A., Meng, X., Solah, V., Devine, A., Binns, C. W., and Prince, R. L. (2015). Consumption of a whey protein-enriched diet may prevent hepatic steatosis associated with weight gain in elderly women. Nutr. Metab. Cardiovas. 25:388-395.

[34] Abd El-Salam MH, El-Shibiny S (2017) Chapter 12-separation of bioactive whey proteins and peptides A2 - Grumezescu, Alexandru Mihai. In: Holban AM (ed) Ingredients extraction by physicochemical methods in food. Academic Press, pp 463-494.

[35] Arihara K, Zhou L, Ohata M (2017) Bioactive properties of Maillard reaction products generated from food protein-derived peptides. Adv Food Nutr Res 81:161-185.

[36] Ashaolu, T.J. and Zhao, G., 2020. Fabricating a Pickering Stabilizer from Okara Dietary Fibre Particulates by Conjugating with Soy Protein Isolate via Maillard Reaction. Foods, 9(2), p.14 